\title{
Intestinal Obstruction and Ileocolic Fistula due to Intraluminal Migration of a Gossypiboma
}

\author{
Evangelos Margonis, ${ }^{1}$ Dionysia Vasdeki, ${ }^{1}$ Alexandros Diamantis, ${ }^{1}$ Georgios Koukoulis, ${ }^{1}$ \\ Grigorios Christodoulidis, ${ }^{1}$ and Konstantinos Tepetes ${ }^{1,2}$ \\ ${ }^{1}$ Department of Surgery, University Hospital of Larissa, Mezourlo, Larissa, 41110 Thessaly, Greece \\ ${ }^{2}$ General Surgery, Faculty of Medicine, University of Thessaly, 41500 Larissa, Greece \\ Correspondence should be addressed to Evangelos Margonis; evangelosmargonis@outlook.com.gr
}

Received 29 November 2015; Revised 28 January 2016; Accepted 1 February 2016

Academic Editor: Cheng-Yu Long

Copyright (C) 2016 Evangelos Margonis et al. This is an open access article distributed under the Creative Commons Attribution License, which permits unrestricted use, distribution, and reproduction in any medium, provided the original work is properly cited.

\begin{abstract}
Gossypiboma refers, as a term, to a retained surgical sponge. It is considered as a rare surgical complication which can occur despite precautions. We report a case of a 36-year-old woman who was admitted to our surgical department with symptoms of abdominal pain associated with episodes of nausea and vomiting that lasted for 2 months. Six months ago she had undergone a cesarean section in a private clinic. Computed tomography revealed a high-density mass occupying a portion of the intestinal lumen, which was reported as a "calcified parasite." The patient was subjected to laparotomy. The intraoperative findings included signs of obstructive ileus and ileosigmoid fistula and a large sponge was found at the resected portion of the small intestine. Although gossypiboma is a rare entity, it should be included in the differential diagnosis.
\end{abstract}

\section{Introduction}

Gossypiboma is a medical term that has been used to describe a serious postoperative complication. A retained gauze/sponge in the abdominal cavity after a surgical procedure has been mainly related with this entity. Terms that have also been used to describe this situation are textiloma, cottonoid, cottonballoma, or gauzeoma [1]. The term gossypiboma derives from the Latin word "gossypium" and the Swahili word "boma," which mean cotton and a "place of concealment," respectively [2]. The term textiloma originates from the word textile which describes the main material of the innocent foreign bodies (gauzes, sponges, and swabs) that are mainly used intraoperatively. When a gauze is accidentally left in an abdominal cavity, then the patient may experience what has been described as intra-abdominal gossypiboma. Intra-abdominal gossypiboma may present with a variety of gastrointestinal manifestations, depending on the location of the retained gauze in the abdomen, with transmural migration of the gauze being a rare manifestation of it. We present the case of an ileosigmoid fistula and small intestine obstruction secondary to intraluminal migration of a sponge left in the abdominal cavity after a cesarean section.

\section{Case Report}

A 36-year-old woman was admitted to our surgical department with abdominal pain associated with episodes of nausea and vomiting over the last couple of months. She denied any history of fever, while she reported a weight loss of around 6 kilos over the same period. She also mentioned that she had undergone a cesarean section in a private clinic 6 months ago. There were no significant findings from the clinical examination of the abdomen. Before having been reviewed by our team, the patient consulted two different general practitioners. The first one set the diagnosis of viral gastroenteritis, while the second advised her to have a computed tomography (CT) scan of her abdomen. The scan was carried out on outpatient basis and revealed a longitudinal, high-density mass inside the intestinal lumen (Figures 1(a) and 1(b)), which was reported as a "calcified parasite." 


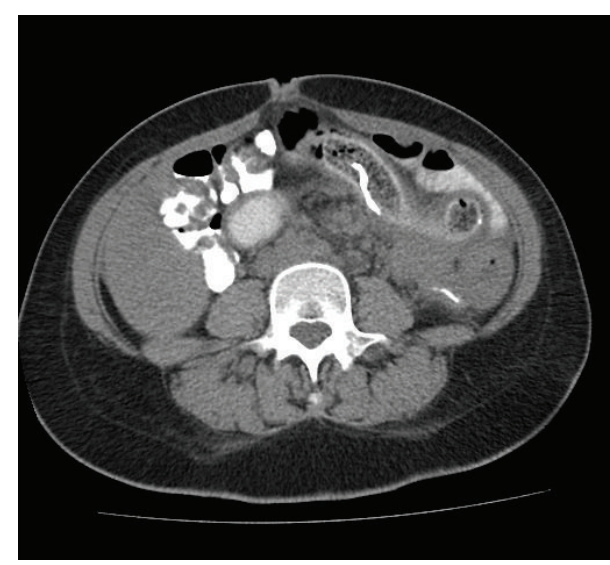

(a)

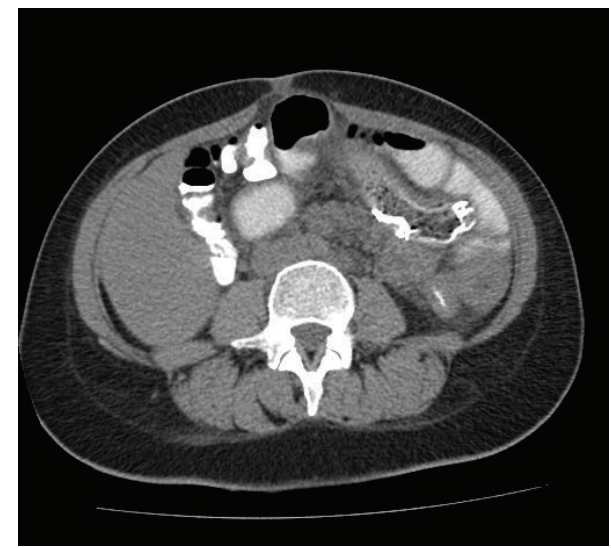

(b)

FIGURE 1: CT images revealing a calcified object inside bowel lumen.

Subsequently, the patient was transferred to our unit. As an inpatient, she had further imaging studies: an abdominal sonography that was normal and a plain abdominal radiograph which revealed a high-density mass in the left iliac fossa mimicking a retained radiopaque body (Figure 2 ).

Blood tests were within normal values. The case was discussed with the patient and her family and a decision was made for the patient to have an explorative laparotomy. Intraoperative findings included dilatation of the small intestine, secondary to obstruction, material in the lumen of an ileal loop, and an ileosigmoid fistula. A small bowel resection en bloc with the affected part of the sigmoid (Figure 3) and a loop sigmoidostomy were carried out. The small intestine was dissected and a $20 \times 25 \mathrm{~cm}$ sponge was found in its lumen (Figures 4(a) and 4(b)). The histological findings are consistent with acute and chronic inflammation and fibrosis of pericolic fat. Liponecrosis is also confirmed, with the presence of histiocytes and multinucleated giant cell producing foreign material phagocytosis.

The patient had an uneventful recovery and was subsequently discharged on the eighth postoperative day. Two months later the patient had a successful restoration of the bowel continuity following a minimally invasive closure of the loop sigmoidostomy.

\section{Discussion}

The first case of a retained cotton-matrix item, a gossypiboma, was described by Wilson in 1884 [3]. The abdominal cavity is the most common site where these masses can be found [4]. There have been described several cases of foreign bodies left in the breast, the nervous system, and the thorax [5]. Although a surgical sponge is the most common retained material in the abdomen following a laparotomy, doctor glasses, light bulbs, porcupine quills, and soft drink bottles have also been reported to have been left in the abdominal cavity [6]. The incidence of gossypiboma varies between 1 in 1000 to 1500 of all intra-abdominal operations and 1 in 100 and 1 in 3000 for all surgical procedures [1, 7-9].

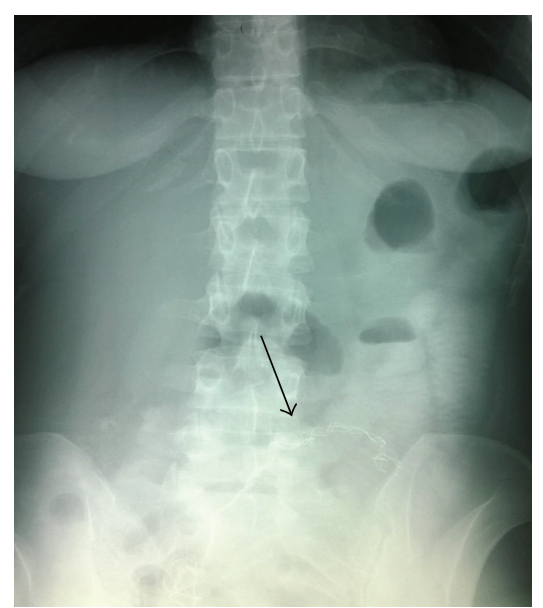

FIGURE 2: Plain abdominal radiograph revealing a gauze in abdominal cavity.

Although this frequency is more likely to be underestimated, the fear of adverse publicity and legal implications leads to underreporting of diagnosed gossypibomas [10]. Serious medicolegal problems may arise between the patient and the surgeon, when a diagnosis of gossypiboma has been set. This conflict may have variable consequences for the surgeon, such as wide negative media coverage, humiliation, mental agony, loss of reputation, and litigation [11]. A retained gauze in the abdominal cavity can cause two types of foreign body reaction: either an exudative reaction, causing an abscess formation, or a foreign body granuloma created by an aseptic fibrinous response [12]. Transmural migration of a gauze is rarely reported in the literature and can lead to fistula formation with adherent organs or even intestinal obstruction. The foreign body migration is attributed to the pressure of the necrosed bowel wall, which has been caused by an encapsulated segmental bowel loop. This encapsulation is the result of the inflammatory reaction of the peritoneum due to the presence of foreign material $[12,13]$. Small bowel and mainly ileum loops are the most commonly affected 


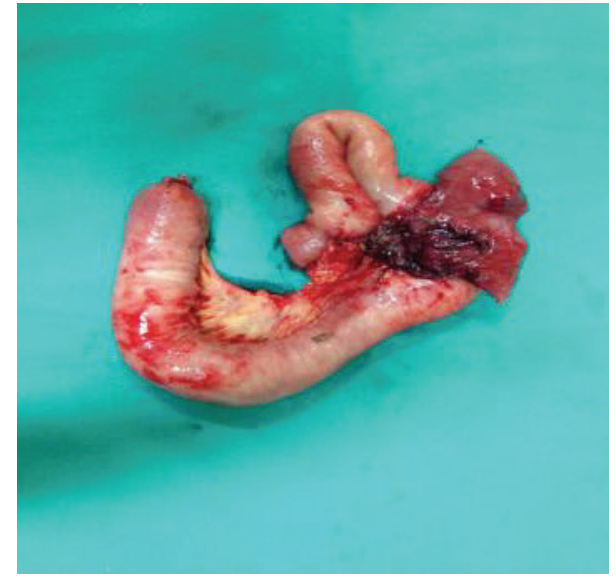

FIGURE 3: Image of the small bowel resection en bloc with part of affected sigmoid colon.

parts of the gastrointestinal tract, while there have been reported a few cases of colon and stomach involvement [7]. Cholecystectomy has been most commonly associated with transmural migration cases, followed by cesarean section and hysterectomy [7]. Preventing the retention of a foreign body is much more crucial than curing the condition which is associated with its retention. Sponge counting protocols must be strictly followed in every operating room. In most hospitals one or two members of the operating team, more usually the nurses, routinely perform a proper sponge counting. All materials and equipment should be counted once at the beginning of an operation and twice at the end of it. The surgeon must explore the abdominal cavity or any other operation site and should never close the wound if an incorrect sponge count exists. Although preventing through counting has been suggested to be the "best treatment," it has been described that most cases occurred although a correct sponge/gauze count was performed. Up to $88 \%$ of reported cases with retained material were associated with a correct surgical material counting before wound closure [14]. Furthermore the use of radioopaque sponges has been related with a reduction in the incidence of this entity and their use should be encouraged [15].

\section{Conclusion}

Intra-abdominal gossypiboma is a rare postoperative complication with potentially severe gastrointestinal manifestations and in most cases the diagnosis may be delayed. The intraluminal location of the retained body has been extremely rarely reported, especially as a cause of intestinal obstruction. Prevention measures and precautions should be our primary goal to avoid such an event, which is associated with significant morbidity and medicolegal implications. The possibility of an existing gossypiboma should always be kept in the physician's mind, when a patient presents with abdominal pain following a recent abdominal surgery.

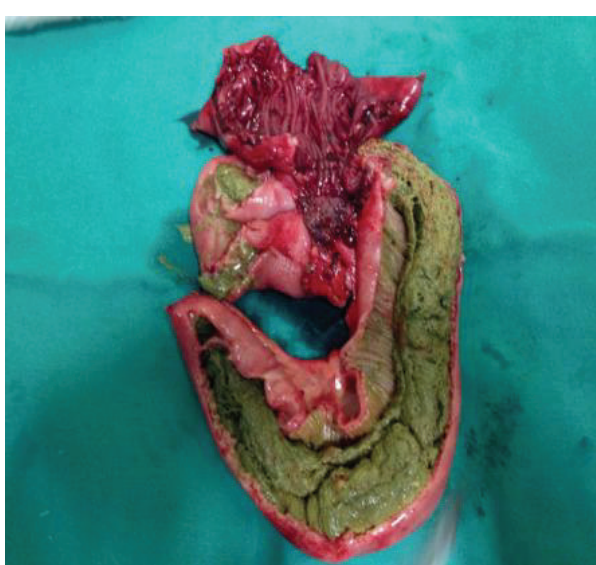

(a)

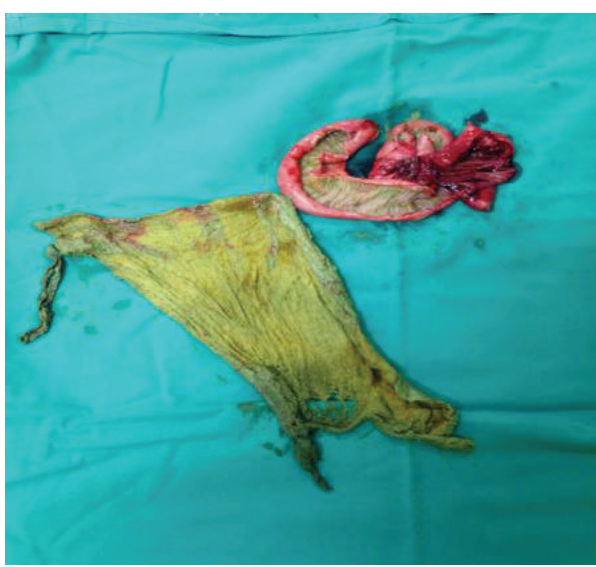

(b)

FIGURE 4: Images of the resected specimen showing the retained foreign body.

\section{Consent}

A written informed consent from the patient for publishing the case report and images is available for review by the editor of this journal.

\section{Conflict of Interests}

No conflict of interests is declared by the authors of this case report.

\section{References}

[1] S. C. Sistla, A. Ramesh, V. S. Karthikeyan, D. Ram, S. M. Ali, and R. V. S. Subramaniam, "Gossypiboma presenting as coloduodenal fistula-report of a rare case with review of literature," International Surgery, vol. 99, no. 2, pp. 126-131, 2014.

[2] K. N. Srivastava and A. Agarwal, "Gossypiboma posing as a diagnostic dilemma: a case report and review of the literature," Case Reports in Surgery, vol. 2014, Article ID 713428, 3 pages, 2014.

[3] C. P. Wilson, "Foreign bodies left in the abdomen after laparotomy," Transactions of the American Gynecological Society, vol. 9, pp. 109-112, 1884. 
[4] B. B. Dash, R. Mahey, A. Kriplani, N. Agarwal, and N. Bhatla, "Textiloma, a rare pelvic tumor," Archives of Gynecology and Obstetrics, vol. 282, no. 6, pp. 707-709, 2010.

[5] A. Manzella, P. B. Filho, E. Albuquerque, F. Farias, and J. Kaercher, "Imaging of gossypibomas: pictorial review," American Journal of Roentgenology, vol. 193, no. 6, supplement, pp. S94-S101, 2009.

[6] H. A. Wells Jr., B. H. Hyun, and R. E. Mitchell Jr., "A retained surgical sponge with interesting sequelae," The American Journal of Medicine, vol. 35, no. 3, pp. 423-426, 1963.

[7] Y.-X. Lv, C.-C. Yu, C.-F. Tung, and C.-C. Wu, "Intractable duodenal ulcer caused by transmural migration of gossypiboma into the duodenum - a case report and literature review," $B M C$ Surgery, vol. 14, article 36, 2014.

[8] A. E. Lincourt, A. Harrell, J. Cristiano, C. Sechrist, K. Kercher, and B. T. Heniford, "Retained foreign bodies after surgery," Journal of Surgical Research, vol. 138, no. 2, pp. 170-174, 2007.

[9] C. W. Kaiser, S. Friedman, K. P. Spurling, T. Slowick, and H. A. Kaiser, "The retained surgical sponge," Annals of Surgery, vol. 224, no. 1, pp. 79-84, 1996.

[10] T. Uluçay, M. G. Dizdar, M. SunayYavuz, and M. Aşirdizer, "The importance of medico-legal evaluation in a case with intraabdominal gossypiboma," Forensic Science International, vol. 198, no. 1-3, pp. e15-e18, 2010.

[11] R. S. Biswas, S. Ganguly, M. L. Saha, S. Saha, S. Mukherjee, and A. Ayaz, "Gossypiboma and surgeon-current medicolegal aspect-a review," Indian Journal of Surgery, vol. 74, no. 4, pp. 318-322, 2012.

[12] A. Sozutek, S. Yormaz, H. Kupeli, and B. Saban, “Transgastric migration of gossypiboma remedied with endoscopic removal: a case report," BMC Research Notes, vol. 6, no. 1, article 413, 4 pages, 2013.

[13] K. K. Patil, S. K. Patil, K. P. Gorad, A. H. Panchal, S. S. Arora, and R. P. Gautam, "Intraluminal migration of surgical sponge: gossypiboma," Saudi Journal of Gastroenterology, vol. 16, no. 3, pp. 221-222, 2010.

[14] M. A. Mahran, E. Toeima, and E. P. Morris, "The recurring problem of retained swabs and instruments," Best Practice \& Research Clinical Obstetrics \& Gynaecology, vol. 27, no. 4, pp. 489-495, 2013.

[15] C. K. Christian, M. L. Gustafson, E. M. Roth et al., "A prospective study of patient safety in the operating room," Surgery, vol. 139, no. 2, pp. 159-173, 2006. 


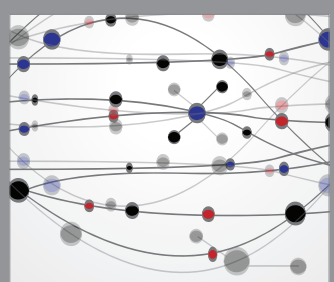

The Scientific World Journal
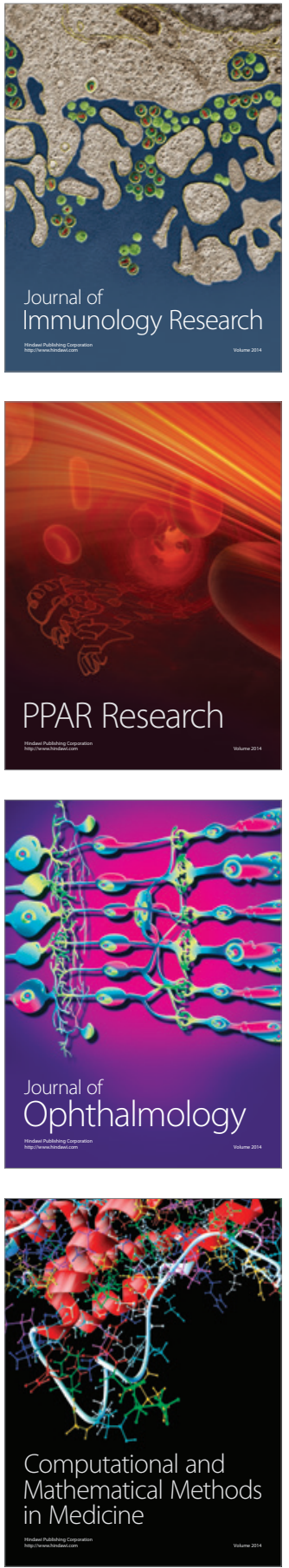

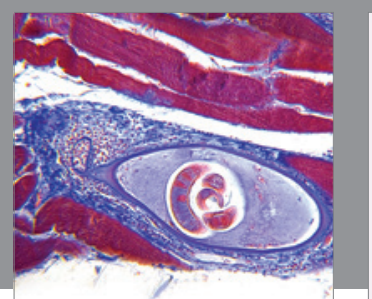

Gastroenterology Research and Practice

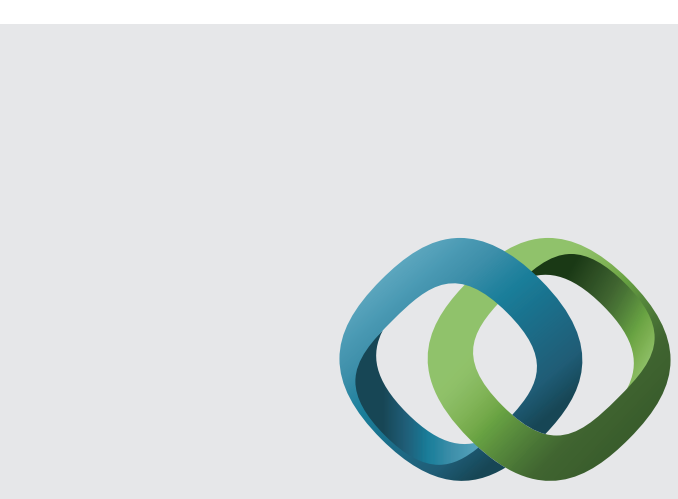

\section{Hindawi}

Submit your manuscripts at

http://www.hindawi.com
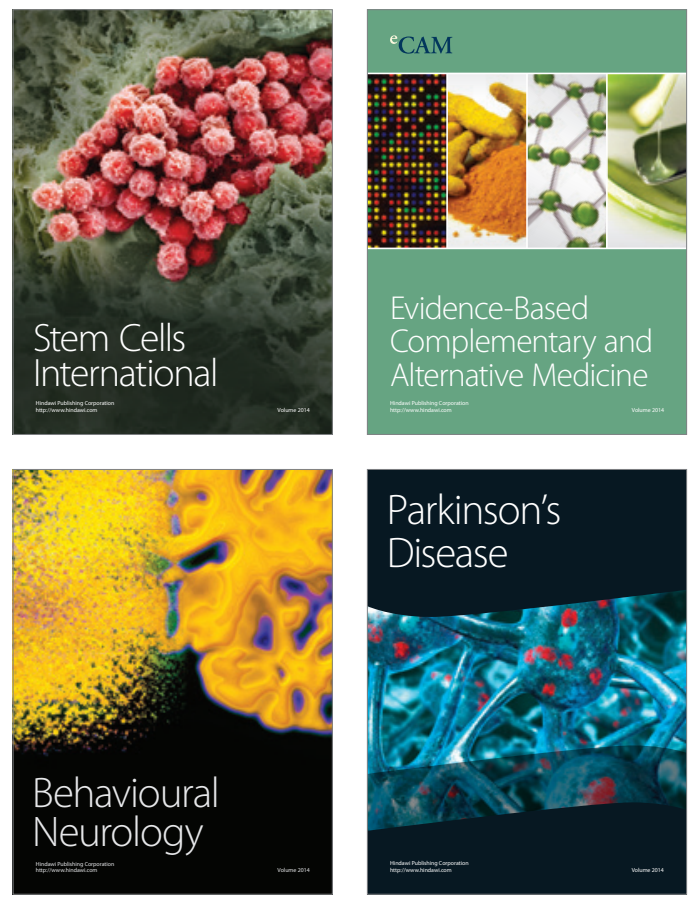
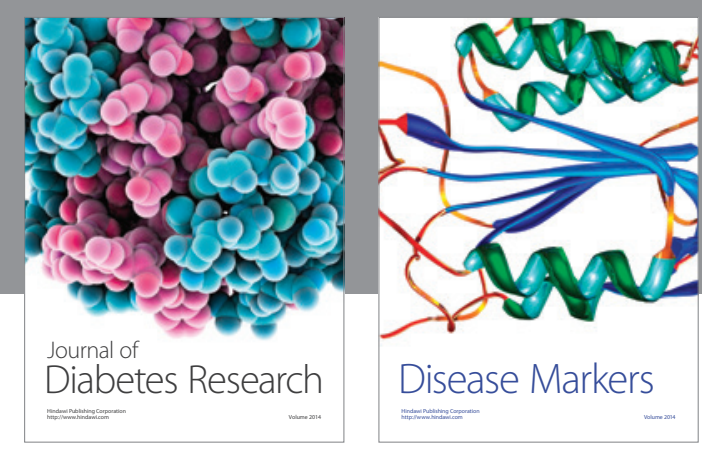

Disease Markers
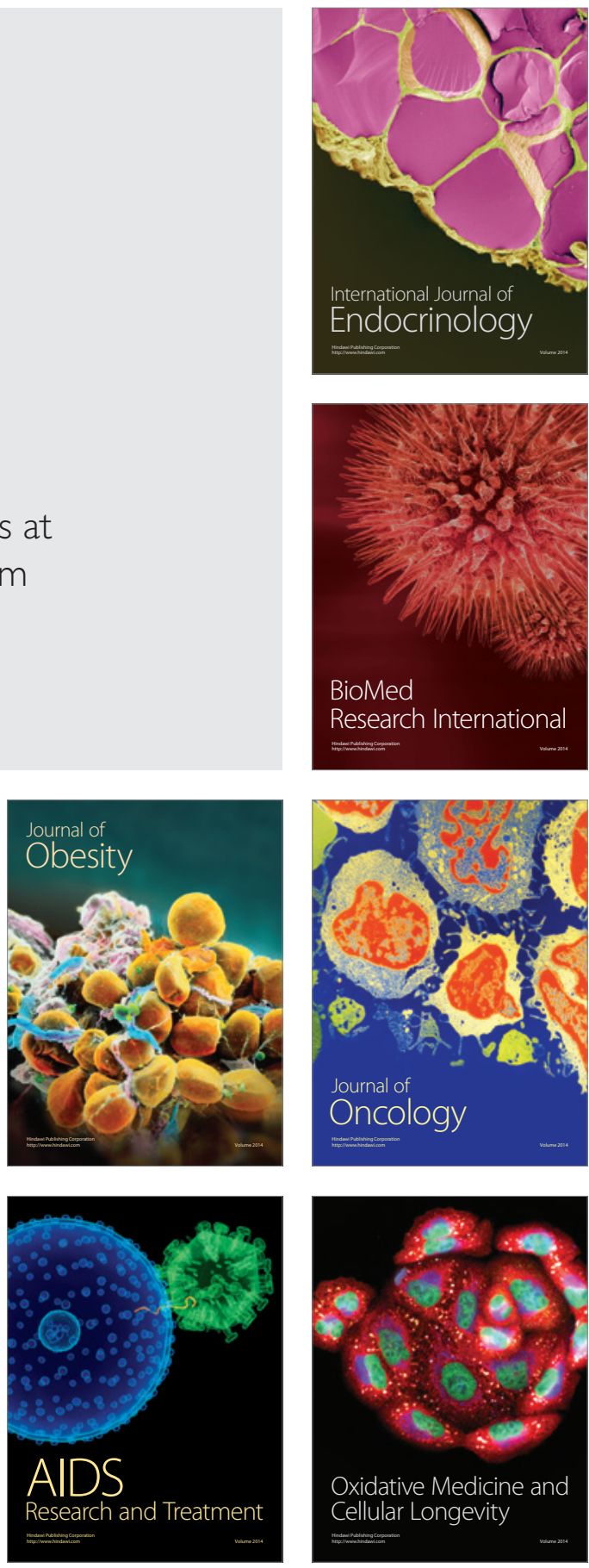